\title{
The Impact of the Implementation of a Surgical Antibiotic Use Guideline in the Practice of Antibiotic Use in the Department of Surgery, Philippine General Hospital
}

\author{
Marie Carmela M. Lapitan, MD, ${ }^{1,2}$ Brian S. Buckley, PhD, ${ }^{2}$ Esther A. Saguil, MD, ${ }^{2}$ Regina P. Berba, MD, ${ }^{3}$ \\ Marissa M. Alejandria, MD, MSc ${ }^{1,3}$ and Zoe Caitlin Z. Fernandez, MD ${ }^{2}$ \\ ${ }^{1}$ Institute of Clinical Epidemiology, National Institutes of Health, University of the Philippines Manila \\ ${ }^{2}$ Department of Surgery, College of Medicine and Philippine General Hospital, University of the Philippines Manila \\ ${ }^{3}$ Department of Medicine, College of Medicine and Philippine General Hospital, University of the Philippines Manila
}

\begin{abstract}
Objective. This study aimed to assess compliance with current best practice guidelines on the use of antibiotics in the Department of Surgery in the Philippine General Hospital and to determine the impact of the dissemination of an institution-based guideline on compliance and on patient outcomes.

Methods. Two antibiotic use surveys were performed 4 weeks before and 4 weeks after the implementation and dissemination of the PGH Surgical Antibiotic Use Guidelines in the Department of Surgery. The medical records of eligible patients were reviewed regarding patient and case characteristics, details on the administration of antibiotics and the collection of specimen for culture studies. Data relating to the occurrence of surgical site infection within 30 days of the operation was extracted. Compliance with antibiotic use guidelines was assessed for each case. The compliance rates in the pre- and post-intervention periods were compared.
\end{abstract}

Results. The study included a total of 477 patients, 213 in the pre-implementation and 264 in the postimplementation period. Compared with the pre-intervention period, rates of compliance with guidelines improved for all parameters in the post-implementation period except for correct dosing. The greatest improvement was seen in the selection of the recommended drug, and proper duration. There was modest improvement in the timing of the preoperative drug administration. There was poor compliance with recommendations for appropriate specimen collection for culture studies, with marked improvement in collection in the pediatric group postintervention. Overall, the in-hospital SSI rate was reduced from $6.8 \%$ to $1.1 \%$, while there was little change in the 30-day SSI rate, post-intervention.

Conclusion. A simple intervention to raise awareness of institutional guidelines on antibiotic use in the surgical setting lead to a modest improvement on overall compliance, although rates of total compliance with all relevant guidance on antibiotic use, choice, dose, timing and duration remained low. The impact on surgical site infection rates based on such compliance was modest.

Key Words: antibiotics, surgery, compliance

\section{BACKGROUND}

Paper presented at the Department of Surgery Staff Conference, May 22, 2019, Philippine General Hospital.

Corresponding author: Marie Carmela M. Lapitan, MD

Department of Surgery

Philippine General Hospital

University of the Philippines Manila

Taft Avenue, Manila 1000, Philippines

Email: mmlapitan@up.edu.ph
Since their first use, antibiotics have become a fundamental part of medicine and have saved millions of lives. However, microbial infections are again emerging as a global threat to human health due to growing resistance of microbes to the antibiotics that were designed to address them. ${ }^{1,2}$ This leads to previously avoidable morbidity and mortality and increased healthcare expenditure due to pro- 
longed hospital stays, protracted and costlier treatments, increased service utilization and increased disability. ${ }^{1}$

The overuse and inappropriate use of antibiotics have been identified as major contributing factors in the growth of antibiotic resistance: countries with higher antibiotic consumption levels have higher incidence of antibiotic resistance ${ }^{1,3-6}$ Levels of consumption of specific antibiotics are associated with the incidence of resistance in the bacteria that they target. ${ }^{3,4}$

The World Health Organization Global Action Plan on antimicrobial resistance, adopted by the World Health Assembly in 2015, proposed strategies for monitoring and controlling the rise of antibiotic resistance. In particular, it highlights the importance of "stewardship programmes that monitor and promote optimization of antimicrobial use at national and local levels in accordance with international standards in order to ensure the correct choice of medicine at the right dose on the basis of evidence." 6

In surgery, the prophylactic use of antibiotics is a cornerstone of infection prevention. International and national guidelines on rational and effective use seek to avoid both excessive and inappropriate antibiotic use. Optimal prophylaxis includes administering the correct antibiotic, the correct dose, the correct time, the correct route, and for the correct duration. ${ }^{7-9}$ However, studies showed that poor compliance with guidelines in many countries were common. ${ }^{10-21}$

This study aimed to assess compliance with current best practice guidelines on the use of antibiotics in a range of surgical procedures in the Department of Surgery in a government tertiary teaching hospital in the Philippines, and to determine the impact of the dissemination of an institution-based guideline on compliance and on patient outcomes.

\section{METHODS}

This study was a quality improvement, before-and-after study conducted among selected cases of the Department of Surgery of the Philippine General Hospital (PGH). Two surveys on antibiotic use were conducted 4 weeks before and 4 weeks after the implementation and dissemination of the PGH Surgical Antibiotic Use Guidelines the Department of Surgery at its staff conference held on February 20, 2019.

The operative and admission logs of the different surgical divisions were reviewed to identify eligible cases for inclusion into the study. The most commonly performed surgical procedures covered by the Department of Surgery guidelines were eligible for inclusion in the survey: appendectomy (simple and complicated), hernia surgery (elective/emergency), complicated intra-abdominal infection, wide excision of tumor, breast surgery (mastectomy with or without reconstruction), thyroid surgery, head and neck surgery (non-thyroid), upper GI surgery, cholangitis, cholecystitis, cholecystectomy, pancreatic surgery, hepatic surgery, small bowel surgery (obstructed/non-obstructed), colorectal surgery(obstructed/non-obstructed), closure of stoma, radical prostatectomy, radical cystectomy, elective cardiac surgery, elective thoracic non-cardiac surgery, elective vascular surgery (elective), and cleft lip and palate repair. Both adult and pediatric cases were included.

The following cases were excluded: patients under an antibiotic regimen for a non-surgical indication at the time of admission; cases with medical conditions that require additional hospitalization and treatment apart from the surgical condition; outpatient cases and cases managed entirely at the emergency room; trauma patients with multiple injuries; patients undergoing several procedures (covering more than one category in the guidelines); patients undergoing eligible surgical procedures but who were admitted in the private service or in another department of the hospital.

The medical records of eligible patients were reviewed and data were extracted regarding patient and case characteristics, details on the administration of antibiotics (including the specific drug used, the dose, timing of the first dose and the preoperative dose, frequency and duration), and the collection of specimen for culture studies. Data relating to the occurrence of surgical site infection (SSI) within 30 days of the operation was extracted for those patients whose procedures were eligible for assessment under the ongoing Department of Surgery Expanded Surgical Site Infection Surveillance Program.

Compliance with antibiotic use guidelines was assessed for each case according to Table 1 . The reference guideline during the pre-implementation phase was the Philippine Department of Health National Antibiotic Guidelines of 2017 or the 2018 Tokyo Guidelines for cases of cholecystitis and cholangitis. ${ }^{22-23}$ Compliance with timing of the preoperative dose was based on the standard of antibiotic administration within one hour prior to start of surgery. ${ }^{24}$ Where no guidance on the duration of antibiotic use has been given in the reference guidelines, compliance was not assessed. The reference guideline during the postimplementation phase assessment was the PGH Surgical Antibiotic Use Guidelines of the Department of Surgery (version February 2019).

The specimen collection rate was determined, based on whether the appropriate specimen was collected for culture studies or not, as recommended in the guidelines. The overall rate and the rate per type of operation requiring specimen collection were computed.

The compliance and specimen collection rates in the pre- and post-intervention periods were compared. Subgroup analysis of compliance rates was done by type of operation/case when at least 5 cases were included per period, and by patient age group (pediatric versus adult). 


\section{RESULTS}

\section{Patient characteristics, pre- and post-imple- mentation}

We included 477 patients, 213 in the pre-implementation and 264 in the post-implementation period. There were slightly more females in the study population, and the majority were adults, with pediatric patients comprising only $15 \%$ of the total population. The most common surgical cases in both periods were appendectomy (simple and complicated), breast surgery particularly elective modified radical mastectomy (MRM), colorectal cancer surgery, elective cholecystectomy and cholangitis. (Table 2).

\section{Comparison of compliance rates, pre and post- implementation, total study population}

Compared with the pre-intervention period, rates of compliance with guidelines improved for all parameters in the post-implementation period except for correct dosing. The greatest improvement was seen in the selection of the recommended drug, and proper duration. There was modest improvement in the timing of the preoperative drug administration (Table 3).
In both pre- and post-intervention periods, compliance with the relevant guidelines was highest for the use of antibiotic prophylaxis when indicated and for use of the correct antibiotic and dose. Compliance was lowest for intraoperative re-dosing of antibiotics when indicated, the proper timing of administration of the preoperative antibiotic and the duration of antibiotics.

Although there was a marked improvement following the intervention, overall compliance, with antibiotic use, type, dose, timing and duration in line with all guidance relevant for each type of surgery, was low for both periods.

\section{Forms of non-compliance}

All instances of non-compliance with guidance on whether antibiotics should be used involved use of antibiotics in cases where they were not recommended. Although compliance with dosing guidance was generally high, nearly all instances of non-compliance involved using doses higher than recommended. Nearly all of the cases that were noncompliant with guidance on duration involved prolonged antibiotic use (Table 4).

Nearly all non-compliance with timing guidance, both for first dose and preoperative dose, involved antibiotic

Table 1. Assessment of compliance to antibiotic use guidelines

\begin{tabular}{|c|c|c|}
\hline \multirow{2}{*}{ Parameter } & \multicolumn{2}{|c|}{ Assessment } \\
\hline & Compliant & Non-compliant \\
\hline $\begin{array}{l}\text { Use of antibiotics (whether } \\
\text { antibiotics were given to the } \\
\text { patient or not, regardless of } \\
\text { the type, dose, duration) }\end{array}$ & $\begin{array}{l}\text { - Used, when recommended } \\
\text { - Not used, if not recommended }\end{array}$ & $\begin{array}{l}\text { - Not used, recommended } \\
\text { - Used, but not recommended }\end{array}$ \\
\hline Drug used & - Type of AB used as per guideline & $\begin{array}{l}\text { - Type of } A B \text { used different from recommended by } \\
\text { the guideline }\end{array}$ \\
\hline Dose & $\begin{array}{l}\text { - Dose of } A B \text { as per guideline } \\
\text { - Dose of } A B \text { is different from guideline but } \\
\text { adjusted as clinically indicated }\end{array}$ & - Dose of $A B$ ordered/given different from guideline \\
\hline First dose timing & $\begin{array}{l}\text { - } A B \text { administered in accordance with timing } \\
\text { recommended in relevant guidelines } \\
\text { - If recommendation is to start therapeutic } A B \\
\text { at diagnosis, } A B \text { must be administered within } \\
1 \text { hour of diagnosis } \\
\text { - If recommendation is to start prophylactic } A B \\
\text { at the time of surgery, } A B \text { must be administered } \\
\text { within the } 60 \text { minutes prior to start of surgery }\end{array}$ & $\begin{array}{l}\text { - } A B \text { administered after the recommended } \\
\text { window (late) } \\
\text { - } A B \text { administered earlier than recommended (early) }\end{array}$ \\
\hline Preoperative dosing & $\begin{array}{l}\text { - AB administered within } 60 \text { minutes prior to } \\
\text { start of surgery }\end{array}$ & $\begin{array}{l}\text { - } \mathrm{AB} \text { administered earlier than } 60 \text { minutes (early) } \\
\text { - } \mathrm{AB} \text { administered after start of surgery (late) } \\
\text { - No preoperative } \mathrm{AB} \text { given }\end{array}$ \\
\hline Duration & - Duration of $A B$ administration as per guideline & $\begin{array}{l}\text { - Duration of } A B \text { administration less than } \\
\text { recommended (short) } \\
\text { - Duration of } A B \text { administration longer than } \\
\text { recommended (prolonged) }\end{array}$ \\
\hline Redosing & $\begin{array}{l}\text { - } A B \text { at the right time and right dose } \\
\text { intraoperatively, as recommended } \\
\text { - No redosing of } A B \text { intraoperatively when } \\
\text { not recommended }\end{array}$ & $\begin{array}{l}\text { - } A B \text { not given when indicated (no redosing) } \\
\text { - Wrong dose given (wrong dose) } \\
\text { - } A B \text { at the wrong time (wrong time) }\end{array}$ \\
\hline Overall & - Compliant in all relevant parameters* & - Non-compliance in at least 1 relevant parameter* \\
\hline
\end{tabular}

${ }^{*}$ Where there was no guidance on the duration of antibiotic use in the reference guidelines, compliance was not assessed.

$A B$, antibiotic 
Table 2. Patient characteristics, pre and post-implementation

\begin{tabular}{|c|c|c|c|}
\hline & $\begin{array}{c}\text { Total } \\
N=477\end{array}$ & $\begin{array}{l}\text { Pre-implementation } \\
\qquad \mathrm{N}=213\end{array}$ & $\begin{array}{l}\text { Post-implementation } \\
\qquad \mathrm{N}=264\end{array}$ \\
\hline $\begin{array}{l}\text { Age - mean (SD) } \\
\text { Pediatric ( } \leq 18 \text { years) } \\
\text { Adult }\end{array}$ & $\begin{array}{c}39.8(18.8) \\
74 \\
404 \\
\end{array}$ & $\begin{array}{c}39.6(18.3) \\
29 \\
185 \\
\end{array}$ & $\begin{array}{c}40.0(19.2) \\
45 \\
219 \\
\end{array}$ \\
\hline $\begin{array}{l}\text { Gender } \\
\text { Male } \\
\text { Female }\end{array}$ & $\begin{array}{l}223 \\
255\end{array}$ & $\begin{array}{r}98 \\
116 \\
\end{array}$ & $\begin{array}{l}125 \\
139 \\
\end{array}$ \\
\hline Operation / Diagnosis & & & \\
\hline $\begin{array}{l}\text { Appendectomy } \\
\text { Simple appendectomy (Lap and Open) } \\
\text { Appendectomy for complicated appendicitis }\end{array}$ & $\begin{array}{r}175 \\
103 \\
72 \\
\end{array}$ & $\begin{array}{l}89 \\
50 \\
39 \\
\end{array}$ & $\begin{array}{r}106 \\
53 \\
33 \\
\end{array}$ \\
\hline $\begin{array}{l}\text { Hernia surgery } \\
\text { Elective hernioplasty } \\
\text { Incarcerated hernia } \\
\text { Strangulated hernia }\end{array}$ & $\begin{array}{r}32 \\
12 \\
18 \\
2\end{array}$ & $\begin{array}{r}16 \\
8 \\
6 \\
2\end{array}$ & $\begin{array}{r}16 \\
4 \\
12 \\
0\end{array}$ \\
\hline $\begin{array}{l}\text { Breast surgery } \\
\text { Mastectomy - clean } \\
\text { Mastectomy - dirty }\end{array}$ & $\begin{array}{r}76 \\
71 \\
5\end{array}$ & $\begin{array}{r}32 \\
29 \\
3\end{array}$ & $\begin{array}{r}44 \\
42 \\
2\end{array}$ \\
\hline Thyroid surgery & 29 & 12 & 17 \\
\hline Complicated intra-abdominal infection & 5 & 3 & 2 \\
\hline $\begin{array}{l}\text { Colorectal surgery } \\
\text { Non-obstructed } \\
\text { Obstructed }\end{array}$ & $\begin{array}{l}57 \\
36 \\
21\end{array}$ & $\begin{array}{l}22 \\
12 \\
10\end{array}$ & $\begin{array}{l}35 \\
24 \\
11 \\
\end{array}$ \\
\hline Closure of stoma & 9 & 4 & 5 \\
\hline Elective simple cholecystectomy (lap and open) & 37 & 9 & 28 \\
\hline Cholangitis & 19 & 8 & 11 \\
\hline Cholecystitis & 13 & 6 & 7 \\
\hline Pancreatic surgery & 6 & 5 & 1 \\
\hline Radical prostatectomy & 2 & 0 & 2 \\
\hline Cleft lip and palate & 7 & 1 & 6 \\
\hline $\begin{array}{l}\text { Wide excision of tumor } \\
\text { Clean } \\
\text { Clean - contaminated } \\
\text { Dirty }\end{array}$ & $\begin{array}{l}9 \\
5 \\
0 \\
4\end{array}$ & $\begin{array}{l}6 \\
3 \\
0 \\
3\end{array}$ & $\begin{array}{l}3 \\
2 \\
0 \\
1\end{array}$ \\
\hline
\end{tabular}

administration earlier than recommended, while the vast majority of non-compliance with re-dosing guidance involved failure to re-dose.

\section{Comparison of compliance rates, pre and post- implementation, according to patient age groups}

Generally, compliance was higher for adult cases, particularly in the drug used, the first dose and the timing of preoperative dose, and duration. Compliance with re-dosing guidance was higher for the pediatric cases (Table 5).

\section{Comparison of compliance rates, pre and post- implementation, according to surgery type}

Compliance rates for operations of which there were at least five during both study periods highlighted differences in prophylactic practices between procedures, which were generally consistent between pre- and post-intervention periods (Table 6). The patterns of compliance by parameters for appendectomy, cholecystitis, cholangitis and colorectal surgery cases broadly reflected compliance in the total population. Compliance with guidelines for some other procedures differed: for example, compliance rates for nearly all parameters were high for clean mastectomy cases and elective hernioplasty, while all thyroidectomy cases were given antibiotics despite the lack of any recommendation to do so.

\section{Appropriate collection of specimen for culture studies}

In general, there was poor compliance with recommendations for appropriate specimen collection for culture studies. There was marked improvement in the collection of tissue specimen in the pediatric group in the post-implementation phase, while there was a decline in the adult cases (Table 7).

\section{In-hospital and 30-day SSI rates, per operation, per survey period.}

Data on the occurrence of surgical site infection was available for 342 of 477 cases ( $72.1 \%$ overall; $162 / 213$, 76\% pre-implementation; $180 / 264,68.9 \%$ post-implementation). Overall, the in-hospital SSI rate was reduced from $6.8 \%$ to $1.1 \%$, while there was little change in the 30 -day SSI 
Table 3. Comparison of compliance rates between pre- and post-implementation periods

\begin{tabular}{lcc|}
\multirow{2}{*}{\multicolumn{1}{c}{ Parameter }} & \multicolumn{2}{c|}{ Compliance Rate } \\
\cline { 2 - 3 } & $\begin{array}{c}\text { Pre-Implementation } \\
\%(\mathrm{n} / \mathrm{N})\end{array}$ & $\begin{array}{c}\text { Post-Implementation } \\
\%(\mathrm{n} / \mathrm{N})\end{array}$ \\
\hline Antibiotic use $^{1}$ & $90.1(192 / 213)$ & $92.4(244 / 264)$ \\
Antibiotic type $^{2}$ & $79.2(152 / 192)$ & $87.5(211 / 241)^{3}$ \\
Dose $^{4}$ & $90.8(138 / 152)$ & $84.4(178 / 211)$ \\
First dose timing $^{2}$ & $66.7(128 / 192)$ & $68.9(168 / 244)$ \\
Pre-op dose timing $^{2}$ & $46.4(89 / 192)$ & $53.0(129 / 244)$ \\
Duration of antibiotics $^{5}$ & $41.1(65 / 158)$ & $64.0(126 / 197)$ \\
Intra-op re-dosing $^{6}$ & $4.6(3 / 66)$ & $5.3(3 / 57)$ \\
Overall compliance $^{7}$ & $11.3(24 / 213)$ & $21.6(57 / 264)$ \\
\hline
\end{tabular}

$A B$, antibiotics

${ }^{1}$ Proportion of surgeries in which ABs were used when recommended.

${ }^{2}$ Denominator is number of cases in which $A B s$ were recommended and used

${ }^{3}$ Three cases not included: $A B$ selection based on culture in 1 case and on allergy to the recommended $A B$ in 2 cases

${ }^{4}$ Denominator is number of cases in which $A B s$ were recommended and the correct type used

${ }^{5}$ Denominator is number of cases in which $A B s$ were recommended and used and for which guidance existed on duration

${ }^{6}$ Denominator is number of cases for which intraoperative $A B$ re-dosing was recommended

${ }^{7}$ Proportion of surgeries in which $A B$ use was in compliance with all relevant guidance

rate. Operations with increased 30-day SSI rates postimplementation included simple appendectomy and clean mastectomy. A decreased SSI rate was noted among cases of colorectal surgery for obstruction (Table 8).

\section{DISCUSSION}

This study determined the effect of a simple intervention to raise awareness of institutional guidelines on compliance with guidelines on antibiotic use in surgery at a large tertiary teaching hospital in the Philippines.

There was total compliance with all relevant guidance on antibiotic use, choice, dose, timing and duration in only $11.3 \%$ of cases in the pre-intervention period. This is in line with a previously published study conducted in the same institution in which $13 \%$ of 244 observed cases conformed to guidelines for all parameters of prophylaxis. ${ }^{25}$ Following the intervention, total compliance rose to $21.6 \%$. Studies worldwide have found that full compliance with relevant
Table 4. Forms of non-compliance, pre- and post-implementation period

\begin{tabular}{|c|c|c|}
\hline \multirow{2}{*}{$\begin{array}{c}\text { Form of } \\
\text { non-compliance }\end{array}$} & \multicolumn{2}{|c|}{ Survey Period } \\
\hline & $\begin{array}{c}\text { Pre-Implementation } \\
\%(n / N)\end{array}$ & $\begin{array}{c}\text { Post-Implementation } \\
\%(n / N)\end{array}$ \\
\hline $\begin{array}{l}\text { Antibiotic use } \\
\text { Used when not } \\
\text { indicated }\end{array}$ & $100(21 / 21)$ & $100(20 / 20)$ \\
\hline $\begin{array}{l}\text { Not used when } \\
\text { indicated }\end{array}$ & 0 & 0 \\
\hline $\begin{array}{l}\text { Drug } \\
\text { Wrong drug used }\end{array}$ & $100(40 / 40)$ & $100(30 / 30)$ \\
\hline $\begin{array}{l}\text { Dose } \\
\text { Under dosed } \\
\text { Over dosed }\end{array}$ & $\begin{array}{c}0 \\
100(14 / 14) \\
\end{array}$ & $\begin{array}{c}12.1(4 / 33) \\
87.8(29 / 33) \\
\end{array}$ \\
\hline $\begin{array}{l}\text { Timing of first dose } \\
\text { Early } \\
\text { Late } \\
\text { Not given } \\
\text { Not indicated } \\
\text { Non-compliant, delayed }\end{array}$ & $\begin{array}{c}82.8(53 / 64) \\
4.7(3 / 64) \\
3.1(2 / 64) \\
4.7(3 / 64) \\
4.7(3 / 64) \\
\end{array}$ & $\begin{array}{c}80.3(61 / 76) \\
13.2(10 / 76) \\
2.6(2 / 76) \\
0(0 / 76) \\
3.9(3 / 76) \\
\end{array}$ \\
\hline Timing of preoperative $c$ & lose & \\
\hline $\begin{array}{l}\text { Earlier than } 60 \text { mins } \\
\text { After incision } \\
\text { Not given }\end{array}$ & $\begin{array}{c}94.1(96 / 112) \\
2.9(3 / 112) \\
2.9(3 / 112) \\
\end{array}$ & $\begin{array}{c}87.0(100 / 115) \\
8.7(10 / 115) \\
4.3(5 / 115) \\
\end{array}$ \\
\hline $\begin{array}{l}\text { Duration } \\
\text { Shorter } \\
\text { Prolonged } \\
\end{array}$ & $\begin{array}{l}10.8(10 / 93) \\
89.2(83 / 93) \\
\end{array}$ & $\begin{array}{l}18.3(13 / 71) \\
81.6(58 / 71) \\
\end{array}$ \\
\hline $\begin{array}{l}\text { Redosing } \\
\text { Not given } \\
\text { Given at a shorter } \\
\text { interval than } \\
\text { recommended }\end{array}$ & $\begin{array}{c}82.2(51 / 62) \\
3.2(2 / 62)\end{array}$ & $\begin{array}{c}85.2(46 / 54) \\
1.9(1 / 54)\end{array}$ \\
\hline $\begin{array}{l}\text { Given at a longer } \\
\text { interval than } \\
\text { recommended }\end{array}$ & $14.5(9 / 62)$ & $13.0(7 / 54)$ \\
\hline
\end{tabular}

guidelines for prophylactic antibiotic use in surgery varies in different locations, but is generally low. Full compliance was reported in $36 \%$ of 898 general surgery cases in a Greek study, $48 \%$ of 100 pediatric surgeries in USA, $7 \%$ of pediatric surgery cases in Israel, and $2 \%$ of abdominal, orthopedic, and gynecological surgeries in Palestine. ${ }^{14,15,17,21}$

In the current study, compliance was high in both preand post-intervention periods with guidance on whether antibiotics should be used at all, and on drug choice and dosage. High compliance with guidance on choice of

Table 5. Compliance rates in pediatric and adult cases, pre and post-implementation period

\begin{tabular}{|c|c|c|c|c|}
\hline \multirow[b]{2}{*}{ Parameter } & \multicolumn{2}{|c|}{ Compliance Rate, Pediatric cases } & \multicolumn{2}{|c|}{ Compliance Rate, Adult cases } \\
\hline & $\begin{array}{c}\text { Pre-Implementation } \\
\%(\mathrm{n} / \mathrm{N})\end{array}$ & $\begin{array}{c}\text { Post-Implementation } \\
\%(\mathrm{n} / \mathrm{N})\end{array}$ & $\begin{array}{c}\text { Pre-Implementation } \\
\%(\mathrm{n} / \mathrm{N})\end{array}$ & $\begin{array}{c}\text { Post-Implementation } \\
\%(n / N)\end{array}$ \\
\hline Use of antibiotics & $100.0(29 / 29)$ & $100.0(45 / 45)$ & $88.6(163 / 184)$ & $90.9(199 / 219)$ \\
\hline Drug used & $69.0(20 / 29)$ & $16.7(6 / 36)$ & $81.0(132 / 163)$ & $82.3(175 / 196)$ \\
\hline Dose & $30.0(6 / 20)$ & $16.7(6 / 36)$ & $70.6(132 / 132)$ & $98.3(172 / 175)$ \\
\hline Timing of first dose & $41.4(12 / 29)$ & $68.9(31 / 45)$ & $71.2(116 / 163)$ & $68.8(137 / 139)$ \\
\hline Timing of preoperative dose & $10.3(3 / 29)$ & $24.4(11 / 45)$ & $52.8(86 / 163)$ & $59.3(118 / 199)$ \\
\hline Duration & $24.1(7 / 29)$ & $47.8(11 / 23)$ & $45.0(58 / 129)$ & $66.1(115 / 174)$ \\
\hline Redosing & $0.0(0 / 2)$ & $0.0(0 / 1)$ & $4.7(3 / 64)$ & $5.4(3 / 56)$ \\
\hline Overall & $0.0(0 / 29)$ & $2.2(1 / 45)$ & $13.0(24 / 184)$ & $25.6(56 / 219)$ \\
\hline
\end{tabular}


Table 6. Compliance rates, per operation (with at least 5 events per period), pre- and post-implementation periods

\begin{tabular}{|c|c|c|}
\hline \multirow{2}{*}{ Parameter } & \multicolumn{2}{|c|}{ Compliance Rate } \\
\hline & $\begin{array}{l}\text { Pre-Implementation } \\
\%(\mathrm{n} / \mathrm{N})\end{array}$ & $\begin{array}{c}\text { Post-Implementatio } \\
\%(\mathrm{n} / \mathrm{N})\end{array}$ \\
\hline \multicolumn{3}{|l|}{ A. Simple appendectomy } \\
\hline Antibiotic prophylaxis & $100.0(50 / 50)$ & $100.0(53 / 53)$ \\
\hline Antibiotic type & $84.0(42 / 50)$ & $94.3(50 / 53)$ \\
\hline Dose & $92.9(39 / 42)$ & $86.0(43 / 50)$ \\
\hline Timing of first dose & $8.0(4 / 50)$ & $7.6(4 / 53)$ \\
\hline $\begin{array}{l}\text { Timing of pre-operative } \\
\text { dose }\end{array}$ & $34.0(17 / 50)$ & $30.2(16 / 53)$ \\
\hline Duration of antibiotics & $38.0(19 / 50)$ & $58.5(31 / 53)$ \\
\hline Intra-operative re-dosing & $18.2(2 / 11)$ & $0.0(0 / 7)$ \\
\hline Overall compliance & $4.0(2 / 50)$ & $0.0(0 / 53)$ \\
\hline \multicolumn{3}{|c|}{ B. Appendectomy for complicated appendicitis } \\
\hline Antibiotic prophylaxis & $100.0(39 / 39)$ & $100.0(33 / 33)$ \\
\hline Antibiotic type & $71.8(28 / 39)$ & $78.8(26 / 33)$ \\
\hline Dose & $43.6(17 / 28)$ & $38.5(10 / 26)$ \\
\hline Timing of first dose & $97.4(38 / 39)$ & $97.0(32 / 33)$ \\
\hline $\begin{array}{l}\text { Timing of pre-operative } \\
\text { dose }\end{array}$ & $25.6(10 / 39)$ & $18.2(6 / 33)$ \\
\hline Duration of antibiotics & $20.5(8 / 39)$ & $0.0(0 / 1)$ \\
\hline Intra-operative re-dosing & $7.1(1 / 14)$ & $28.6(2 / 7)$ \\
\hline Overall compliance & $0.0(0 / 39)$ & $3.0(1 / 33)$ \\
\hline \multicolumn{3}{|l|}{ C. Mastectomy clean } \\
\hline Antibiotic prophylaxis & $100.0(29 / 29)$ & $100.0(42 / 42)$ \\
\hline Antibiotic type & $100.0(29 / 29)$ & $100.0(42 / 42)$ \\
\hline Dose & $100.0(29 / 29)$ & $100.0(42 / 42)$ \\
\hline Timing of first dose & $93.1(27 / 29)$ & $95.2(40 / 42)$ \\
\hline $\begin{array}{l}\text { Timing of pre-operative } \\
\text { dose }\end{array}$ & $93.1(27 / 29)$ & $95.2(40 / 42)$ \\
\hline Duration of antibiotics & $96.6(28 / 29)$ & $95.2(40 / 42)$ \\
\hline Intra-operative re-dosing & $0.0(0 / 9)$ & $0.0(0 / 6)$ \\
\hline Overall compliance & $65.6(19 / 29)$ & $81.0(34 / 42)$ \\
\hline \multicolumn{3}{|l|}{ D. Thyroid surgery } \\
\hline Antibiotic prophylaxis & $0.0(0 / 12)$ & $0.0(0 / 17)$ \\
\hline Antibiotic type & NA & NA \\
\hline Dose & NA & NA \\
\hline Timing of first dose & NA & NA \\
\hline $\begin{array}{l}\text { Timing of pre-operative } \\
\text { dose }\end{array}$ & NA & NA \\
\hline Duration of antibiotics & NA & NA \\
\hline Intra-operative re-dosing & NA & NA \\
\hline Overall compliance & NA & NA \\
\hline \multicolumn{3}{|l|}{ E. Cholecystitis } \\
\hline Antibiotic prophylaxis & $100.0(6 / 6)$ & $100.0(7 / 7)$ \\
\hline Antibiotic type & $83.3(5 / 6)$ & $85.7(6 / 7)$ \\
\hline Dose & $100.0(5 / 5)$ & $100.0(6 / 6)$ \\
\hline Timing of first dose & $100.0(6 / 6)$ & $100.0(7 / 7)$ \\
\hline $\begin{array}{l}\text { Timing of pre-operative } \\
\text { dose }\end{array}$ & $16.7(1 / 6)$ & $14.3(1 / 7)$ \\
\hline Duration of antibiotics & $0.0(0 / 6)$ & $57.1(4 / 7)$ \\
\hline Intra-operative re-dosing & NA & NA \\
\hline Overall compliance & $0.0(0 / 6)$ & $14.3(1 / 7)$ \\
\hline \multicolumn{3}{|l|}{ F. Cholangitis } \\
\hline Antibiotic prophylaxis & $100.0(8 / 8)$ & $100.0(11 / 11)$ \\
\hline Antibiotic type & $62.5(5 / 8)$ & $72.7(8 / 11)$ \\
\hline Dose & $100.0(5 / 5)$ & $100.0(8 / 8)$ \\
\hline Timing of first dose & $100.0(8 / 8)$ & $100.0(11 / 11)$ \\
\hline $\begin{array}{l}\text { Timing of pre-operative } \\
\text { dose }\end{array}$ & $0.0(0 / 8)$ & $0.0(0 / 11)$ \\
\hline Duration of antibiotics & $50.0(4 / 8)$ & $0.0(0 / 11)$ \\
\hline Intra-operative re-dosing & NA & NA \\
\hline Overall compliance & $0.0(0 / 8)$ & $22.2(2 / 9)$ \\
\hline
\end{tabular}

\begin{tabular}{|c|c|c|}
\hline \multirow{2}{*}{ Parameter } & \multicolumn{2}{|c|}{ Compliance Rate } \\
\hline & $\begin{array}{c}\text { Pre-Implementation } \\
\%(n / N)\end{array}$ & $\begin{array}{c}\text { Post-Implementation } \\
\%(n / N)\end{array}$ \\
\hline \multicolumn{3}{|c|}{ G. Elective cholecystectomy } \\
\hline Antibiotic prophylaxis & $33.3(3 / 9)$ & $100.0(28 / 28)$ \\
\hline Antibiotic type & $100.0(3 / 3)$ & $92.9(26 / 28)$ \\
\hline Dose & $100.0(3 / 3)$ & $100.0(26 / 26)$ \\
\hline Timing of first dose & $33.3(1 / 3)$ & $78.6(22 / 28)$ \\
\hline $\begin{array}{l}\text { Timing of pre-operative } \\
\text { dose }\end{array}$ & $33.3(1 / 3)$ & $78.6(22 / 28)$ \\
\hline Duration of antibiotics & $66.7(2 / 3)$ & $63.0(17 / 27)$ \\
\hline Intra-operative re-dosing & $0.0(0 / 2)$ & $0.0(0 / 9)$ \\
\hline Overall compliance & $0.0(0 / 9)$ & $42.9(12 / 28)$ \\
\hline \multicolumn{3}{|l|}{ H. Elective hernioplasty } \\
\hline Antibiotic prophylaxis & $100.0(8 / 8)$ & $100.0(4 / 4)$ \\
\hline Antibiotic type & $75.0(6 / 8)$ & $75.0(3 / 4)$ \\
\hline Dose & $100.0(6 / 6)$ & $33.3(1 / 3)$ \\
\hline Timing of first dose & $100.0(8 / 8)$ & $100.0(4 / 4)$ \\
\hline $\begin{array}{l}\text { Timing of pre-operative } \\
\text { dose }\end{array}$ & $100.0(8 / 8)$ & $100.0(4 / 4)$ \\
\hline Duration of antibiotics & $25.0(2 / 8)$ & $100.0(4 / 4)$ \\
\hline Intra-operative re-dosing & NA & NA \\
\hline Overall compliance & $12.5(1 / 8)$ & $25.0(1 / 4)$ \\
\hline \multicolumn{3}{|c|}{ I. Emergency hernioplasty (Incarcerated / Strangulated) } \\
\hline Antibiotic prophylaxis & $100.0(8 / 8)$ & $100.0(12 / 12)$ \\
\hline Antibiotic type & $25.0(2 / 8)$ & $33.3(4 / 12)$ \\
\hline Dose & $100.0(2 / 2)$ & $25.0(1 / 4)$ \\
\hline Timing of first dose & $50.0(4 / 8)$ & $8.3(1 / 12)$ \\
\hline $\begin{array}{l}\text { Timing of pre-operative } \\
\text { dose }\end{array}$ & $37.5(3 / 8)$ & $25.0(3 / 12)$ \\
\hline Duration of antibiotics & $16.7(1 / 6)$ & $75.0(9 / 12)$ \\
\hline Intra-operative re-dosing & $0.0(0 / 3)$ & $0.0(0 / 2)$ \\
\hline Overall compliance & $0.0(0 / 8)$ & $0.0(0 / 12)$ \\
\hline \multicolumn{3}{|c|}{ J. Colorectal surgery (non-obstructed) } \\
\hline Antibiotic prophylaxis & $100.0(12 / 12)$ & $100.0(24 / 24)$ \\
\hline Antibiotic type & $91.7(11 / 12)$ & $100.0(24 / 24)$ \\
\hline Dose & $66.7(8 / 12)$ & $100.0(24 / 24)$ \\
\hline Timing of first dose & $66.7(8 / 12)$ & $91.7(22 / 24)$ \\
\hline $\begin{array}{l}\text { Timing of pre-operative } \\
\text { dose }\end{array}$ & $0.0(0 / 10)$ & $91.7(22 / 24)$ \\
\hline Duration of antibiotics & NA & $63.6(14 / 22)$ \\
\hline Intra-operative re-dosing & $0.0(0 / 10)$ & $0.0(0 / 16)$ \\
\hline Overall compliance & $8.3(1 / 12)$ & $58.3(14 / 24)$ \\
\hline \multicolumn{3}{|c|}{ K. Colorectal surgery (obstructed) } \\
\hline Antibiotic prophylaxis & $100.0(10 / 10)$ & $100.0(11 / 11)$ \\
\hline Antibiotic type & $80.0(8 / 10)$ & $72.3(8 / 11)$ \\
\hline Dose & $100.0(8 / 8)$ & $100.0(8 / 8)$ \\
\hline Timing of first dose & $100 / 0(10 / 10)$ & $81.8(9 / 11)$ \\
\hline $\begin{array}{l}\text { Timing of pre-operative } \\
\text { dose }\end{array}$ & $40.0(4 / 10)$ & $27.3(3 / 11)$ \\
\hline Duration of antibiotics & NA & $0.0(0 / 11)$ \\
\hline Intra-operative re-dosing & $0.0(0 / 7)$ & $14.3(1 / 7)$ \\
\hline Overall compliance & $0.0(0 / 10)$ & $0.0(0 / 11)$ \\
\hline
\end{tabular}


Table 7. Specimen culture rates, by age group, in pre- and post-implementation period

\begin{tabular}{|c|c|c|c|c|}
\hline \multirow{3}{*}{$\begin{array}{l}\text { Culture } \\
\text { Specimen }\end{array}$} & \multicolumn{4}{|c|}{ Collection rates } \\
\hline & \multicolumn{2}{|c|}{ Adult } & \multicolumn{2}{|c|}{ Pediatric } \\
\hline & $\begin{array}{l}\text { Pre-implementation } \\
\%(n / N)\end{array}$ & $\begin{array}{c}\text { Post-implementation } \\
\%(\mathrm{n} / \mathrm{N})\end{array}$ & $\begin{array}{c}\text { Pre-implementation } \\
\%(\mathrm{n} / \mathrm{N})\end{array}$ & $\begin{array}{l}\text { Post-implementation } \\
\%(n / N)\end{array}$ \\
\hline Tissue & $13.3(11 / 83)$ & $11.6(8 / 69)$ & $16.7(2 / 12)$ & $63.6(14 / 22)$ \\
\hline Blood & $63.6(7 / 11)$ & $46.2(6 / 13)$ & NA & NA \\
\hline Bile & $7.1(1 / 14)$ & $5.88(1 / 17)$ & NA & $0(0 / 1)$ \\
\hline
\end{tabular}

Table 8. SSI rates, in-hospital and 30-day, in pre- and post-implementation

\begin{tabular}{|c|c|c|c|c|}
\hline \multirow{3}{*}{ Case } & \multicolumn{4}{|c|}{ SSI rates } \\
\hline & \multicolumn{2}{|c|}{ In-hospital SSI rate } & \multicolumn{2}{|c|}{ 30-day SSI rate } \\
\hline & $\begin{array}{c}\text { Pre-implementation } \\
\%(n / N)\end{array}$ & $\begin{array}{c}\text { Post-implementation } \\
\%(\mathrm{n} / \mathrm{N})\end{array}$ & $\begin{array}{l}\text { Pre-implementation } \\
\%(\mathrm{n} / \mathrm{N})\end{array}$ & $\begin{array}{c}\text { Post-implementation } \\
\%(\mathrm{n} / \mathrm{N})\end{array}$ \\
\hline Simple appendectomy & $2.1(1 / 48)$ & $2.1(1 / 48)$ & $8.3(4 / 48)$ & $16.7(8 / 48)$ \\
\hline Appendectomy for complicated appendicitis & $7.9(3 / 38)$ & $0.0(0 / 31)$ & $21.1(8 / 38)$ & $22.6(7 / 31)$ \\
\hline Mastectomy (clean) & $0.0(0 / 29)$ & $0.0(0 / 41)$ & $0.0(0 / 29)$ & $7.3(3 / 41)$ \\
\hline Mastectomy (dirty) & $66.7(2 / 3)$ & $0.0(0 / 2)$ & $100.0(3 / 3)$ & $50.0(1 / 2)$ \\
\hline Colorectal surgery (obstructed) & $22.2(2 / 9)$ & $0.0(0 / 9)$ & $44.4(4 / 9)$ & $22.2(2 / 9)$ \\
\hline Colorectal surgery (non-obstructed) & $0.0(0 / 12)$ & $0.0(0 / 23)$ & $0.0(0 / 12)$ & $4.4(1 / 23)$ \\
\hline Closure of stoma & $0.0(0 / 4)$ & $0.0(0 / 4)$ & $0.0(0 / 4)$ & $0.0(0 / 4)$ \\
\hline Pancreatic Surgery & $25.0(1 / 4)$ & $100(1 / 1)$ & $25.0(1 / 4)$ & $100(1 / 1)$ \\
\hline Elective Cholecystectomy & $33.3(1 / 3)$ & $0(0 / 6)$ & $33.3(1 / 3)$ & $16.7(1 / 6)$ \\
\hline Cholangitis & $0(0 / 6)$ & $0(0 / 9)$ & $0(0 / 6)$ & $22.2(2 / 9)$ \\
\hline Cholecystitis & $16.7(1 / 6)$ & $0(0 / 6)$ & $16.7(1 / 6)$ & $0(0 / 6)$ \\
\hline Total & $6.8(11 / 162)$ & $1.1(2 / 180)$ & $13.6(22 / 162)$ & $15.0(27 / 180)$ \\
\hline
\end{tabular}

SSI, Surgical site infection

antibiotic is in line with studies in USA and Israel that both observed correct antibiotic choice in $97 \%$ of cases, and in Greece and Qatar where rates of 70\% and 68\%, respectively were observed. ${ }^{10,14,15,21}$ Levels of compliance with dosing guidance in both periods was comparable with rates reported by studies in USA and Israel (77\% and 52\%, respectively.)

Compliance was much poorer with guidance on timing of both first dose and of preoperative dose and on duration of antibiotic use, although there was some improvement following the intervention. Compliance with guidance on timing fall within a wide range of adherence rates reported by studies from Greece (100\%), Australia (93\%), USA (73\%), Palestine (60\%) and Israel (32\%). ${ }^{13-15,17,21}$ Similarly, compliance with guidance on duration was in the mid-range of adherence rates ranging from $88 \%$ to $32 \%$ reported by other studies. ${ }^{10,13,15,17,21}$ Compliance was the lowest with guidance on intraoperative re-dosing, with this not being done at all in the majority of cases in which it was indicated.

Given the known associations between antibiotic resistance and the overuse and inappropriate use of antibiotics, it is noted in this study that much of the noncompliance with guidance was on whether antibiotics should be used at all, and overuse (dosing and duration). During the two study periods, antibiotics were used in 41 cases in which they were not indicated, 43 cases received higher doses than recommended, and in 141 cases, antibiotics were administered for longer durations than recommended. This is an issue that is widespread in many health care settings: it has been estimated that treatment indication, choice of antibiotic and duration of administration is incorrect in $30-50 \%$ of cases. ${ }^{1,2,26}$ In the current study there was low compliance with recommendations on specimen collection for culture studies. Again, this is an issue that is not uncommon in many health care settings. Pressure of time, the way health systems are organized and the accessibility of pathology services can lead to failure to identify pathogens. In one study, a causative pathogen was identified in less than $8 \%$ of patients hospitalized with community-acquired pneumonia in the US. ${ }^{27}$

Although hospitals account for a small proportion of total antibiotic prescribing in humans, the hospital setting is one in which issues relating to antibiotic use and resistance are brought sharply into focus. In hospitals, per capita rates of prescribing are higher than in other settings, the incidence of detected antimicrobial resistance is highest in hospitals, the health of patients is already compromised and hospital-acquired infections are common. Consequently, clinicians struggle daily to identify antibiotics that can help patients with resistant infections. ${ }^{28-29}$

This study has several strengths, such as good data availability and a relatively large number of cases. However, several limitations are acknowledged. Data were limited to relatively short time periods, and for selected types of procedures. Factors that could have affected compliance were not considered by the study. And the study was not powered to detect associations between guidance compliance and the incidence of SSI. 
Further research should focus on identifying factors that affect compliance, effective means of improving compliance, and associations between compliance and SSI rates.

\section{CONCLUSION}

A simple intervention to raise awareness of institutional guidelines on antibiotic use in the surgical setting led to a modest improvement on overall compliance. However, rates of total compliance with all relevant guidance on antibiotic use, choice, dose, timing and duration remained low. Further initiatives should be introduced and maintained to improve practice in all parameters of antibiotic use, and to promote appropriate specimen collection for culture studies.

\section{Statement of Authorship}

All authors approved the final version of the submitted paper.

\section{Author Disclosure}

All authors declared no conflicts of interest related to this paper.

\section{Funding Source}

The study was funded by: (1) Dr. Fidel Estrada Research Fund grant, (2) Foundation for the Advancement of Surgical Education, Inc, grant and (3) PGH-Expanded Hospital Research Office Research Grant.

\section{REFERENCES}

1. Centers for Disease Control and Prevention, Office of Infectious Disease. Antibiotic resistance threats in the United States, 2013. Atlanta CDC (Centers for Disease Control and Prevention) [Internet]. 2013 [cited 2018 Oct]. Available from: http://www.cdc. gov/drugresistance/threat-report-2013/index.html

2. Lee Ventola C. The Antibiotic resistance crisis. P T. 2015 Apr; 40(4):277-83.

3. ECDC (European Centre for Disease Prevention and Control), EFSA (European Food Safety Authority), EMA (European Medicines Agency). ECDC/EFSA/EMA second joint report on the integrated analysis of the consumption of antimicrobial agents and occurrence of antimicrobial resistance in bacteria from humans and foodproducing animals. EFSA Journal. 2017; 15(7):4872. doi:10.2903/ j.efsa.2017.4872.

4. ECDC (European Centre for Disease Prevention and Control), EFSA (European Food Safety Authority), EMA (European Medicines Agency). ECDC/EFSA/EMA first joint report on the integrated analysis of the consumption of antimicrobial agents and occurrence of antimicrobial resistance in bacteria from humans and foodproducing animals. EFSA Journal. 2015; 13(1):4006. doi:10.2903/ j.efsa.2015.4006.

5. Goossens H. Antibiotic consumption and link to resistance. Clin Microbiol Infect. 2009 Apr; 15(Suppl 3):12-5. doi: 10.1111/j.14690691.2009.02725.x.

6. World Health Organization. Global Action Plan on Antimicrobial Resistance. Geneva: World Health Organisation [Internet]. 2015 [cited 2018 Oct]. Available from: https://apps.who.int/iris/ handle/10665/193736

7. Surgical site infection: prevention and treatment of surgical site infection. London: National Collaborating Centre for Women's and Children's Health / National Institute for Health and Clinical Excellence. 2008.

8. Bratzler DW, Dellinger EP, Olsen KM, Perl TM, Auwaerter PG, Bolon MK, et al. ASHP Therapeutic Guidelines: Clinical practice guidelines for antimicrobial prophylaxis in surgery. Am J Health Syst Pharm. 2013 Feb; 70(3):195-283. doi: 10.2146/ajhp120568.

9. Scottish Intercollegiate Guidelines Network (SIGN). Antibiotic prophylaxis in surgery. Edinburgh: SIGN [Internet]. 2014 [cited 2018 Oct]. Available from: https://www.just.edu.jo/ar/DIC/Clinic Guidlines/Antibiotic\%20prophylaxis\%20in\%20surgery.pdf

10. Abdel-Aziz A, El-Menyar A, Al-Thani H, Zarour A, Parchani A, Asim M, et al. Adherence of surgeons to antimicrobial prophylaxis guidelines in a tertiary general hospital in a rapidly developing country. Adv Pharmacol Sci. 2013; 2013:842593. doi: 10.1155/ 2013/842593.

11. Andrajati R, Vlcek J, Kolar M, Pipalova R. Survey of surgical antimicrobial prophylaxis in Czech Republic. Pharm World Sci. 2005 Dec; 27(6):436-41. doi: 10.1007/s11096-005-5971-8.

12. Flores AMP, Pena AC, Buenconcejo LS. Adherence of surgeons to the Philippine College of Surgons Clinical Practice Guidelines on Antibiotic Prophylaxis for elective surgical procedures at the Quirino Memorial Medical Center. Philipp J Microbiol Infect Dis. 2004; 33(1):29-34.

13. Friedman ND, Styles K, Gray AM, Low J, Athan E. Compliance with surgical antibiotic prophylaxis at an Australian teaching hospital. Am J Infect Control. 2013 Jan; 41(1):71-4. doi: 10.1016/ j.ajic.2012.02.012.

14. Hawkins RB, Levy SM, Senter CE, Zhao JY, Doody K, Kao LS, et al. Beyond surgical care improvement program compliance: antibiotic prophylaxis implementation gaps. Am J Surg. 2013 Oct; 206(4):451-6. doi: 10.1016/j.amjsurg.2013.02.009.

15. Klinger G, Carmeli I, Feigin E, Freud E, Steinberg R, Levy I. Compliance with surgical antibiotic prophylaxis guidelines in pediatric surgery. Eur J Pediatr Surg. 2015 Apr; 25(2):199-202. doi: 10.1055/s-0034-1368798.

16. Matti PRA, Querol RC, Antonio-Velmonte M, de Vera RL, Alejandria M. Prescribing practices of surgeons and factors that limit adherence to the Philippine College of Surgeons Clinical Practice Guidelines on Antimicrobial Prophylaxis for elective surgical procedures at the UP-PGH surgical wards. Philipp J Microbiol Infect Dis. 2002; 31(3):107-24.

17. Musmar SMJ, Ba'ba H, Owais A. Adherence to guidelines of anti-biotic prophylactic use in surgery: a prospective cohort study in North West Bank, Palestine. BMC Surg. 2014 Sep; 14(69). doi:10.1186/ 471-2482-14-69.

18. Parulekar L, Soman R, Singhal T, Rodrigues C, Dastur FD, Mehta A. How good is compliance with surgical antibiotic prophylaxis guidelines in a tertiary care private hospital in India? A prospective study. Indian J Surg. 2009 Feb; 71(1):15-8. doi: 10.1007/s12262009-0004-9

19. Pons-Busom M, Aguas-Compaired M, Delas J, Eguileor-Partearroyo B. Compliance with local guidelines for antibiotic prophylaxis in surgery. Infect Control Hosp Epidemiol. 2004 Apr; 25(4):308-12. doi: 10.1086/502397.

20. Sagayaga HM, Ampil IDE II. An assessment of compliance of surgical practitioners in a university hospital with Philippine college of surgeons clinical practice guidelines on antimicrobial prophylaxis in elective surgical procedures. Philipp J Surg Spec. 2004; 59(1):29-37.

21. Tourmousoglou CE, Yiannakopoulou EC, Kalopothaki V, Bramis J, Papadopoulos JS. Adherence to guidelines for antibiotic prophylaxis in general surgery: a critical appraisal. J Antimicrob Chemother. 2008 Jan; 61(1):214-8. doi: 10.1093/jac/dkm406.

22. Gomi H, Solomkin JS, Schlossberg D, Okamoto K, Takada T, Strasberg SM, et al. Tokyo Guidelines 2018: antimicrobial therapy for acute cholangitis and cholecystitis. J Hepatobiliary Pancreat Sci. 2018 Jan; 25(1):3-16. doi: 10.1002/jhbp.518.

23. DOH. National Antibiotic Guidelines 2017. Manila: Department of Health, 2017. 
24. WHO. Global Guidelines for the Prevention of Surgical Site Infection. Geneva: World Health Organization [Internet]. 2016 [cited 2018 Oct]. Available from: https://apps.who.int/iris/bitstream/ handle $/ 10665 / 250680 / 9789241549882$-eng.pdf?sequence $=8$

25. Nabor MIP, Buckley BS, Lapitan MCM. Compliance with international guidelines on antibiotic prophylaxis for elective surgeries at a tertiary-level hospital in the Philippines. Healthcare Infection. 2015 Sep; 20(3-4):145-51. doi: 10.1071/HI15018

26. Luyt CE, Brechot N, Trouillet JL, Chastre J. Antibiotic stewardship in the intensive care unit. . Crit Care. 2014 Aug; 18(5):480. doi: 10.1186/s13054-014-0480-6.

27. Bartlett JG, Gilbert DN, Spellberg B. Seven ways to preserve the miracle of antibiotics. Clin Infect Dis. 2013 May; 56(10):1445-50. doi: $10.1093 / \mathrm{cid} / \mathrm{cit} 070$.
28. Joseph NM, Bhanupriya B, Shewade DG, Harish BN. Relationship between antimicrobial consumption and the incidence of antimicrobial resistance in Escherichia coli and Klebsiella pneumoniae isolates. J Clin Diagn Res. 2015 Feb; 9(2):8-12. doi: 10.7860/ JCDR/2015/11029.5537.

29. Meyer E, Gastmeier P, Deja M, Schwab F. Antibiotic consumption and resistance: Data from Europe and Germany. Int J Med Microbiol. 2013 Aug; 303(6-7):388-95. doi: 10.1016/ j.ijmm.2013.04.004.
The Acta Medica Philippina is now accepting original scientific papers, review articles and case reports for its upcoming issues. Please follow the format for submission as indicated in the "Instructions to Authors" elsewhere in the journal. All papers received shall be properly acknowledged. For inquiries and submission of proposals, please email us at actamedicaphilippina.upm@up.edu.ph 